\title{
HORIZONTES DE POSIBILIDAD PARA LA PRODUCCIÓN DE CONOCIMIENTO EN OCIO, RECREACIÓN Y TIEMPO LIBRE EN COLOMBIA ${ }^{1}$
}

\author{
HORIZONS OF POSSIBILITY FOR PRODUCTION OF KNOWLEDGE \\ IN LEISURE, RECREATION AND FREE TIME IN COLOMBIA
}

\author{
José Fernando Tabares Fernández ${ }^{2}$ \\ Víctor Alonso Molina Bedoya ${ }^{3}$
}

Resumen

En este artículo se presentan los resultados de la investigación Producción de Conocimiento en Ocio, Recreación y Tiempo Libre en América Latina, específicamente lo atinente a las condiciones de posibilidad para la generación de conocimiento en Colombia; intención que hace parte de un objetivo mayor cual es el de develar el interés de conocimiento que subyace a las investigaciones en ocio, recreación, tiempo libre y lazer en las producciones científicas de América Latina en el siglo XXI. A nivel metodológico el ejercicio privilegia el abordaje cualitativo de la investigación, con especial interés en el trabajo descriptivo, utilizando el análisis de contenido y la investigación bibliográfica como estrategia de interrogación mediante el análisis sistemático de los documentos. En términos de resultados se constata un efecto importante de las realidades particulares del país en los desarrollos científicos logrados en el campo. Este desarrollo está marcado por la política pública científica y se expresa en la existencia o no de procesos de formación de maestría y doctorado, en la formación de investigadores, la consolidación de los grupos de investigación, las temáticas y el número de investigadores existentes, los eventos académicos realizados en el país y la existencia de revistas especializadas. Así, la pregunta por las condiciones de posibilidad para la generación de conocimiento no es de fácil respuesta, precisa de trabajos articulados y de la generación de redes locales, nacionales e internacionales que permitan posicionar el campo como una oportunidad para el buen vivir y bienestar de los pueblos.

Pallabras clave: Conocimiento, recreación, Colombia, investigación (Decs).

\section{Abstract}

This article presents the results of the research project entitled: Knowledge Production in Leisure, Recreation and Freetime in Latin America, particularly in connection with the conditions that make it possible the generation of new knowledge in Colombia. This purpose is part of a larger goal -to reveal the interest of knowledge underlying research in leisure, recreation and lazer in scientific production in Latin America in the twenty first century. This is a qualitative study, with emphasis in description. Content analysis and library research serve as an inquiry strategy, through a systematic review of literature. In terms of results, there is an important effect of the country's particular realities in the scientific developments achieved in this area. This development is marked by the scientific public policy as expressed in the existence - or lack - of training processes at master's and doctorate's levels, in researchers' training, the makeup of research groups, research topics, and the number of existing researchers, academic events in the country and the publication of specialized journals. Thus, the question for the conditions that make knowledge generation a possibility is not easily answered. It requires a coordinated work and the creation of local, domestic and international networks to position the topic as an opportunity for good life and people's well-being.

Keywords: Knowledge, recreation, Colombia, Research

Fecha de recepción: 15 de julio de 2014

Fecha de aprobación: 12 de noviembre de 2014

1 El artículo se construye en el marco de la investigación internacional: Producción de conocimiento en ocio, tiempo libre y recreación en América Latina.

2 Doctor. Profesor de la Universidad de Antioquia. Miembro del grupo de investigación Ocio, Expresiones Motrices y Sociedad. Correo electrónico: jfernando37@hotmail.com

3 Doctor. Profesor titular de la Universidad de Antioquia. Director del grupo de investigación Ocio, Expresiones Motrices y Sociedad. Correo electrónico: victor.molina@udea.edu.co 
Para citar este artículo:

Tabares, J., Molina, V. (2014). Horizontes de posibilidad para la producción de conocimiento en ocio, recreación y tiempo libre en Colombia. Revista Lúdica Pedagógica, (20), 113-118.

No hay enseñanza sin investigación $n i$ investigación sin enseñanza. Esos quehaceres se encuentran cada uno en el cuerpo del otro. Mientras enseño continúo buscando, indagando. Enseño porque busco, porque indagué, porque indago y me indago. Investigo para comprobar, comprobando intervengo, interviniendo educo y me educo. Investigo para conocer lo que aún no conozco y comunicar o anunciar la novedad.

Paulo Freire (1997, p. 30)

\section{INTRODUCCIÓN}

El estudio expone resultados referidos a las condiciones de posibilidad para la generación de conocimiento en Colombia de la investigación internacional Producción de conocimiento en ocio, recreación y tiempo libre en América Latina; proyecto que se propone develar el interés de conocimiento que subyace a las investigaciones en ocio, recreación, tiempo libre y lazer América Latina en el siglo XXI.

Metodológicamente se privilegió el abordaje cualitativo de la investigación, con especial interés en el trabajo descriptivo, utilizando el análisis de contenido y la investigación bibliográfica como estrategia de interrogación mediante el análisis sistemático de los documentos.

Como principal resultado se destaca el efecto que, sobre la producción científica, tienen las condiciones y relaciones sociales imperantes en los territorios para la generación de conocimiento. Para Lamo de Espinosa et al., como se cita en Brunet y Pastor:

Todos los conocimientos son producidos socialmente y sus sentidos son socialmente contingentes: todos los procesos de producción, validación y cambio del conocimiento científico son el resultado de procesos de interacción social [...] entre científi$\cos [\ldots]$ o entre estos y el medio social circundante [...] (2002, p. 32).

Esto es, los conocimientos producidos en un campo de saber-conocer específico son resultado de la interacción de múltiples factores y condiciones sociales que para un determinado momento histórico se expresan en la política pública científica de un lugar y que, para el caso de la investigación, adquieren forma entre muchos otros asuntos, en la existencia o no de procesos de formación de maestría y doctorado, en la formación de investigadores, la consolidación de los grupos de investigación, las temáticas y número de investigadores existentes, los eventos académicos realizados en el territorio nacional y la existencia de revistas especializadas.

Para el caso de Colombia, aun cuando se tiene una política pública de ciencia y tecnología, el campo del ocio, la recreación y el tiempo libre no goza de los recursos y las condiciones para lograr un buen desarrollo investigativo y de producción científica. El artículo presenta una referencia general a lo que sucede con relación a la ausencia de una estrategia que permita la organización del campo, los programas para la formación del nivel de posgrado, los grupos de investigación y las publicaciones especializadas.

\section{AUSENCIA DE UNA ESTRATEGIA QUE PERMITA LA ORGANIZACIÓN DEL CAMPO}

Una dificultad encontrada por el grupo de investigación para la definición del corpus de trabajo fue la ausencia de una estrategia organizativa nacional que permitiera ubicar la información de los trabajos, las investigaciones y estudios que, sobre el campo, se han realizado en el país. Ante esta situación y luego de una revisión sobre los posibles lugares, se identificó el evento académico nacional que realiza la Fundación Colombiana de Tiempo Libre y Recreación Funlibre, que convoca a diferentes actores e instituciones del campo. Así, los congresos y simposios nacionales de recreación son los más representativos y regulares, pues se organizan desde 1990, y es a partir de su quinta versión, que Funlibre asume su liderazgo.

Sin embargo, llama la atención al equipo de investigación que el liderazgo en términos de socialización y encuentro de los agentes del sector lo lleve una organización privada y no una entidad propia de los actores del campo o una institución del Estado, finalmente responsable de su garantía como derecho que ha sido consignado en la Constitución Política.

Esta dispersión del campo evidencia el escaso impacto que, a cinco años de la ejecución del Plan Decenal del Deporte, la Recreación, la Educación Física y la Actividad Física, para el Desarrollo Humano, la Convivencia y la Paz. 2009- 2019, se ha tenido en la implementación del Registro Único Nacional del Deporte (RUN-SND), cuyo propósito ha sido precisamente ser un instrumento eficaz de registro de las organizaciones relacionadas con el sector. Esto se encuentra claramente 
expuesto en el numeral 5.1 sobre Lineamiento de política 1: Organización y fortalecimiento institucional del sector (Coldeportes Nacional, 2009, p. 19).

Indiscutiblemente, para el caso de la producción de conocimiento en el campo del ocio y la recreación, las dificultades de organización y articulación entre los agentes y las instituciones desfavorecen los trabajos colaborativos para poder actuar como un sistema. Ya para el año de formulación del Plan, se hablaba de problemas de concepción y de autonomía de la recreación para poder ser una estrategia de desarrollo humano (Coldeportes Nacional, 2009, p. 30).

\section{PROGRAMAS PARA LA FORMACIÓN DEL NIVEL DE POSGRADO EN EL CAMPO}

En referencia a los posgrados, uno de los problemas identificados en el campo es la ausencia de programas del nivel de maestría y doctorado. Solo existen programas de especialización. De los seis programas que aparecen registrados ante el Ministerio de Educación Nacional, solo dos están activos en la actualidad (tabla 1). Frente a esta ausencia, las personas se ven obligadas a adelantar sus procesos de formación avanzada en programas de Educación Física, o en campos como la educación, la salud o el desarrollo humano, entre otros. Esta situación

Tabla 1. Programas de Posgrado en Ocio, Recreación y Lúdica en Colombia (snies, 2013).

\begin{tabular}{|c|c|c|c|c|c|c|c|c|}
\hline № & Institución & $\begin{array}{c}\text { Nombre } \\
\text { posgrado }\end{array}$ & $\begin{array}{c}\text { Área de } \\
\text { conocimiento }\end{array}$ & $\begin{array}{c}\text { Nivel de } \\
\text { formación }\end{array}$ & Metodología & Duración & Título & $\begin{array}{c}\text { Situación } \\
\text { actual }\end{array}$ \\
\hline 1 & $\begin{array}{c}\text { Fundación } \\
\text { Universitaria } \\
\text { Juan de } \\
\text { Castellanos }\end{array}$ & $\begin{array}{c}\text { Lúdica } \\
\text { Educativa }\end{array}$ & $\begin{array}{c}\text { Ciencias de la } \\
\text { Educación }\end{array}$ & Especialización & $\begin{array}{c}\text { Distancia } \\
\text { (tradicional) }\end{array}$ & $\begin{array}{c}\text { Dos } \\
\text { semestres }\end{array}$ & $\begin{array}{c}\text { Especialista } \\
\text { en Lúdica } \\
\text { Educativa }\end{array}$ & Activa \\
\hline 2 & $\begin{array}{l}\text { Universidad } \\
\text { de Pamplona }\end{array}$ & $\begin{array}{c}\text { Educación } \\
\text { para la } \\
\text { Recreación } \\
\text { Comunitaria }\end{array}$ & $\begin{array}{c}\text { Ciencias de la } \\
\text { Educación }\end{array}$ & Especialización & Presencial & $\begin{array}{c}\text { Dos } \\
\text { semestres }\end{array}$ & $\begin{array}{c}\text { Especialista } \\
\text { en } \\
\text { Educación } \\
\text { para la } \\
\text { Recreación } \\
\text { Comunitaria }\end{array}$ & Inactiva \\
\hline 3 & $\begin{array}{c}\text { Escuela } \\
\text { Nacional del } \\
\text { Deporte }\end{array}$ & $\begin{array}{c}\text { Dirección } \\
\text { y Gestión } \\
\text { de la } \\
\text { Recreación }\end{array}$ & $\begin{array}{c}\text { Economía, } \\
\text { Administración, } \\
\text { Contaduría, etc. }\end{array}$ & Especialización & Presencial & $\begin{array}{c}\text { Dos } \\
\text { semestres }\end{array}$ & $\begin{array}{c}\text { Especialista } \\
\text { en Dirección } \\
\text { y Gestión } \\
\text { de la } \\
\text { Recreación }\end{array}$ & Inactiva \\
\hline 4 & $\begin{array}{c}\text { Fundación } \\
\text { Universitaria } \\
\text { los } \\
\text { Libertadores }\end{array}$ & $\begin{array}{c}\text { Lúdica y } \\
\text { Recreación } \\
\text { para el } \\
\text { Desarrollo } \\
\text { Cultural } \\
\text { y Social }\end{array}$ & $\begin{array}{l}\text { Ciencias } \\
\text { Sociales y } \\
\text { Humanas }\end{array}$ & Especialización & Presencial & $\begin{array}{l}\text { Cuatro } \\
\text { trimestres }\end{array}$ & $\begin{array}{c}\text { Especialista } \\
\text { en Lúdica y } \\
\text { Recreación } \\
\text { para el } \\
\text { Desarrollo } \\
\text { Social y } \\
\text { comunitario }\end{array}$ & Inactiva \\
\hline 5 & $\begin{array}{c}\text { Fundación } \\
\text { Universitaria } \\
\text { los } \\
\text { Libertadores }\end{array}$ & $\begin{array}{c}\text { Pedagogía } \\
\text { de la } \\
\text { Recreación } \\
\text { Musical } \\
\text { para el } \\
\text { Desarrollo } \\
\text { Social y } \\
\text { Cultural }\end{array}$ & $\begin{array}{c}\text { Ciencias de la } \\
\text { Educación }\end{array}$ & Especialización & $\begin{array}{c}\text { Distancia } \\
\text { (tradicional) }\end{array}$ & $\begin{array}{c}\text { Dos } \\
\text { semestres }\end{array}$ & $\begin{array}{c}\text { Especialista } \\
\text { en } \\
\text { Pedagogía } \\
\text { de la } \\
\text { Recreación } \\
\text { Músical } \\
\text { para el } \\
\text { Desarrollo } \\
\text { Social y } \\
\text { Cultural }\end{array}$ & Activa \\
\hline
\end{tabular}


conduce a la dispersión de la producción, la investigación y las problematizaciones necesarias para el desarrollo del campo.

Los escasos programas de posgrado existentes en el campo pueden ser reflejo de lo que sucede en los pregrados, donde se destaca poca continuidad en los procesos de formación de los niveles técnico, tecnológico y profesional, como sucedió con el Politécnico Colombiano Jaime Isaza Cadavid. En la actualidad, la Universidad del Valle tiene vigente el programa de profesionalización en Recreación, donde los estudiantes tienen la opción de titularse hasta el sexto semestre como tecnólogos y continuar hasta su profesionalización con diez semestres. La Universidad Pedagógica Nacional con su programa de formación de Licenciados en Recreación estructurado a partir de dos ciclos: uno de fundamentación y otro de profundización. Y con fuerte presencia en la actualidad se encuentra el Sena con los programas de Técnico en Recreación y Tecnólogo en Gestión de Servicios Recreativos, con una duración de veinticuatro meses.

No obstante este panorama, es preciso tener presente que muchos profesionales realizan trabajos de investigación y de grado en otras áreas de conocimiento, lo que permite abordajes interdisciplinarios del objeto en cuestión y propicia el avance del campo de saber-conocer.

Para el equipo de investigación es importante el desarrollo de los posgrados ya que permiten profundizar y producir conocimiento en un campo tan complejo e importante en la vida de las personas y los colectivos, como lo es el del ocio y la recreación. El avance en la configuración de maestrías y doctorados permitirá un mayor desarrollo de la investigación y la reflexión sobre el tema, acorde con las necesidades y particularidades de la región y de los países, y a la vez una mejor capacitación de los investigadores para un conocimiento más cualificado y comprometido con el mejoramiento de las formas de vida de la población.

\section{GRUPOS DE INVESTIGACIÓN}

En referencia a los grupos de investigación en la plataforma de Colciencias -entidad responsable de la política de ciencia, tecnología e innovación en el país- aparecen 13 grupos registrados. Solo uno contiene en su denominación la palabra ocio, tres incluyen el descriptor recreación asociado a otros como actividad física, deportes, cultura física, educación física, muy propio de los programas que forman profesionales en el país, y donde la recreación se comprende como un medio de la educación física. En el turismo se inscriben ocho de los grupos y no se relacionan con el ocio, la recreación o el tiempo libre; un grupo aparece con el descriptor animación cultural. Como se observa, en general hay una muy baja presencia de grupos de investigación inscritos en el campo de interés.

Este es quizás uno de los rastros más contundentes en lo que a condiciones de posibilidad para la producción de conocimiento en el campo se refiere. Solo 14 grupos referenciados a temas relacionados, que no dedicados, con la profundización y consolidación de propuestas epistemológicas y metodológicas para el desarrollo del campo en el país. También es importante resaltar la situación de la Universidad Tecnológica de Pereira, donde las líneas Recreación Terapéutica y Recreación y Cotidianidad se encuentran adscritas al grupo Cultura de la Salud, perteneciente a la Facultad de Ciencias de la Salud. Y la Universidad del Valle, con un programa de formación en Recreación, donde existe la línea Recreación, Intersubjetividades e Interculturalidad, perteneciente al Grupo Educación Popular del Instituto de Educación y Pedagogía. Este asunto, junto con los procesos de formación de investigadores, debería recibir un apoyo decidido en el horizonte de la configuración de una comunidad académica fuerte, que desde sus particularidades aporte a la consolidación de procesos de investigación a largo plazo. Un modelo que debe partir del respeto por la diversidad, tanto de prácticas y contextos, como de perspectivas epistemológicas.

Con respecto a la actividad de los grupos, no se cuenta con ejercicios que permitan una lectura de los intereses, las tendencias o las perspectivas teóricas que sustentan su hacer investigativo. Como cualquier otro campo de conocimiento, el ocio y la recreación posee una tradición, un acumulado donde se hacen visibles apuestas epistémicas y formas de realización de la práctica investigativa. Este tipo de ejercicios sería fundamental para generar autorreflexión crítica sobre lo que hacemos, pero sobre todo, para entender la forma de organización y estructuración como campo, esto es, como escenario de encuentros y disputas entre agentes, instituciones y discursos. Como ya se ha hecho en otros países de la región, se encuentran investigaciones que dan cuenta de los objetos de interés de los grupos, de sus perspectivas metodológicas, de sus articulaciones con pares nacionales e internacionales, de las estrategias para crear generación de relevo desde sus propios propósitos de indagación, del tipo de publicaciones que privilegian, de los medios donde divulgan lo que investigan, del tipo de literatura que referencian, de sus autores de cabecera, de la forma como se relaciona con otros agentes del sector y de otros sectores, entre muchos otros asuntos que es necesario problematizar para dar cuenta de la actividad científica en general del país. 
Tabla 2. Grupos de investigación registrados en Colciencias

\begin{tabular}{|c|c|c|c|c|c|c|c|c|}
\hline \multirow[b]{2}{*}{ Descriptor } & \multirow[b]{2}{*}{ Nombre del grupo } & \multirow[b]{2}{*}{ Total } & \multicolumn{6}{|c|}{ Áreas de los grupos con interés en los temas de ocio, recreación y tiempo libre } \\
\hline & & & Educación & $\begin{array}{l}\text { Ciencias } \\
\text { Humanas }\end{array}$ & $\begin{array}{c}\text { Ciencias } \\
\text { Sociales } \\
\text { Aplicadas }\end{array}$ & $\begin{array}{l}\text { Ciencias } \\
\text { Sociales }\end{array}$ & $\begin{array}{l}\text { Ciencias de } \\
\text { la Salud }\end{array}$ & $\begin{array}{l}\text { Educación } \\
\text { Física }\end{array}$ \\
\hline Ocio & $\begin{array}{l}\text { Ocio, Expresiones } \\
\text { Motrices y Sociedad }\end{array}$ & 1 & $\mathrm{X}$ & $\mathrm{x}$ & & & & \\
\hline Lúdica & $\begin{array}{l}\text { Lúdica, Cuerpo } \\
\text { y Sociedad }\end{array}$ & 1 & $\mathrm{X}$ & & & $\mathrm{X}$ & & \\
\hline \multirow{3}{*}{ Recreación } & $\begin{array}{l}\text { Actividad Física, } \\
\text { Recreación y Deporte }\end{array}$ & \multirow{3}{*}{3} & & & & & $\mathrm{x}$ & $\mathrm{X}$ \\
\hline & $\begin{array}{c}\text { Ciencias Sociales, } \\
\text { Cultura Física, } \\
\text { Deporte y Recreación }\end{array}$ & & & & & & $\mathrm{X}$ & $\mathrm{X}$ \\
\hline & $\begin{array}{l}\text { Epistemología } \\
\text { e Historia de la } \\
\text { Educación Física, } \\
\text { la Recreación y el } \\
\text { Deporte -Hierde- }\end{array}$ & & & & & & $\mathrm{X}$ & $\mathrm{x}$ \\
\hline \multirow{8}{*}{ Turismo } & Turismo & \multirow{8}{*}{8} & & & $\mathrm{X}$ & & & \\
\hline & Turismo y Sociedad & & & & $\mathrm{x}$ & & & \\
\hline & $\begin{array}{c}\text { Turismo, Medio } \\
\text { Ambiente y } \\
\text { Desarrollo }\end{array}$ & & & & $\mathrm{X}$ & & & \\
\hline & $\begin{array}{c}\text { Grupo de } \\
\text { Investigación } \\
\text { en Ciencias } \\
\text { Administrativas y } \\
\text { Turismo -Incatur- }\end{array}$ & & & & $\mathrm{X}$ & & & \\
\hline & $\begin{array}{c}\quad \text { Grupo de } \\
\text { Investigación } \\
\text { Empresarial y } \\
\text { Turístico }\end{array}$ & & & & $\mathrm{X}$ & & & \\
\hline & $\begin{array}{c}\text { Desarrollo Turístico } \\
\text { y Regional }\end{array}$ & & & & $\mathrm{X}$ & & & \\
\hline & $\begin{array}{c}\text { Grupo de } \\
\text { Investigación } \\
\text { Impacto del Turismo } \\
\text { Sexual -Giffi- }\end{array}$ & & & & $\mathrm{x}$ & & & \\
\hline & Turismo & & & & $\mathrm{X}$ & & & \\
\hline $\begin{array}{l}\text { Animación } \\
\text { cultural }\end{array}$ & $\begin{array}{c}\text { Grupo de } \\
\text { Investigación } \\
\text { para la Animación } \\
\text { Cultural -Muisuata- }\end{array}$ & 1 & & & $\mathrm{X}$ & & & \\
\hline \multicolumn{2}{|c|}{ Total consolidado } & 14 & 2 & 1 & 9 & 1 & 3 & 3 \\
\hline
\end{tabular}

Fuente: Cálculos propios 


\section{PUBLICACIONES ESPECIALIZADAS}

Sobre revistas especializadas en el tema específico del ocio y la recreación, se encuentra la Revista Latinoamericana de Recreación, editada por Funlibre con dos números a la fecha. También es importante nombrar las ediciones de los Cuadernos de Ocio, liderada por el Instituto de Educación Física de la Universidad de Antioquia, que entre 1997 y 2000 logró editar cinco números. Luego apareció Cuadernos de Ocio y Sociedad, de la Corporación Civitas, que ha publicado tres números, con artículos correspondientes a resultados de investigación y aportes de académicos de otros países de América Latina.

En el sector de la educación física, la recreación y el deporte, aparecen reconocidas en Publindex Colciencias año 2014, las revistas Educación Física y Deporte de la Universidad de Antioquia, categoría B de periodicidad semestral; Lúdica Pedagógica de la Universidad Pedagógica Nacional categoría B, semestral y Actividad Física y Desarrollo Humano de la Universidad de Pamplona, categoría $\mathrm{C}$ con edición anual.

Estas revistas ubican como áreas de interés, a su modo: educación física y deporte, la educación física, el deporte, la recreación, el ocio, el tiempo libre, las ciencias de la actividad física y demás disciplinas relacionadas. Por su parte, Lúdica Pedagógica aborda la educación, la pedagogía, la investigación y la ciencia en los campos de la educación física, la recreación y el deporte; y la revista Actividad Física y Desarrollo Humano es multidisciplinaria.

En términos de las condiciones de posibilidad para la publicación de artículos resultado de investigaciones, reflexiones, ensayos, etc., el panorama del campo en el país no es el mejor, si se tienen en cuenta las nuevas exigencias planteadas por las universidades a sus investigadores de publicar en revistas de alto impacto (A1, A2), dado que para poder publicar en otros campos de conocimiento parece que fuera necesario una subordinación a esos discursos de mayor prestigio, tradición y hegemonía.

\section{CONCLUSIONES}

La pregunta por las condiciones de posibilidad para la generación de conocimiento en cualquier campo no es de fácil respuesta. Para Estela Quintar (2007), una reflexión sobre la producción de conocimiento en la universidad en general implica reflexionar acerca de la formación de los sujetos que lo producen. Para ella, la formación es un proceso sustantivo en la producción de conocimiento, tanto de los que enseñan e investigan, como de quienes aprenden en los distintos campos de la ciencia.

Su abordaje y comprensión precisa de trabajos articulados y de la generación de redes locales, nacionales e internacionales que permitan posicionar el campo como una oportunidad de investigación y de sistematización de conocimiento para el buen vivir y bienestar de los pueblos, congruente con sus dinámicas políticas, económicas y sociales.

Como se ha indicado en el desarrollo del documento, las dificultades tanto de organización como de articulación entre los agentes e instituciones restringe la generación de trabajos colaborativos para efectivamente poder funcionar como sistema.

Es urgente avanzar en la formulación de programas de maestría y doctorado que permitan profundizar y producir conocimiento en un campo tan complejo como el del ocio y la recreación.

Vivir en sociedades que operan bajo modelos de ciencia e investigación centrados en la calidad y en la burocratización demanda de los actores, profesionales e investigadores actitudes críticas y reflexivas sobre lo epistemológico como pensamiento y postura ante la realidad.

\section{REFERENCIAS BIBLIOGRÁFICAS}

Brunet, I. y Pastor, I. (2002). Epistemología o sociología de la ciencia. Revista Praxis Sociológica. 6, 24-40.

Coldeportes Nacional (2009). Plan Decenal del Deporte, la Recreación, la Educación Física y la Actividad Física, para el Desarrollo Humano, La Convivencia y la Paz. 2009- 2019.

Lamo de Espinosa, E. et al. (1994). La sociologia del conocimiento y de La ciencia. Madrid: Alianza.

Publindex, (2014). Recuperado de http://scienti.colciencias.gov.co:8084/publindex/EnArticulo/busqueda.do

Freire, P. (1997). Pedagogía de la autonomía. Saberes necesarios para la práctica educativa. México: Siglo XXI.

Quintar, E. (2007). Universidad, producción de conocimiento y formación en América Latina, Polis [En línea], 18. Recuperado de http://polis.revues. org/4096. 